\title{
Empowering Your Presentation Skills
}

\author{
${ }^{1}$ Michael S Cardwell, ${ }^{2} \mathrm{~J}$ Hector Aranda, ${ }^{3}$ Rene Hernandez, ${ }^{4} \mathrm{Hoi}$ Ho
}

\section{ABSTRACT}

As technology continues evolving, ultrasonography has become increasingly the imaging modality of choice in many different clinical settings including hospital, clinic and point of care. Similarly, the expansion of internet has revolutionized the medical curriculum and training of the entire medical education continuum: undergraduate medical education, graduate medical education and continuing medical education. More importantly, technology and internet have transformed the traditional teacher-dependent classroom-based teaching into the learneroriented web-based learning. To empower the presentation, whether classroom-based or web-based, it's essential that faculty members apply principles of adult learning throughout the session. Newer version of PowerPoint (PPT) is powerful and user-friendly. However, faculty members should be familiar with fundamental guidelines for appropriate selection of fonts, character sizes, background colors, charts, graphs, animation and multimedia.

In general, PowerPoint presentations prepared on Windowsbased computers can be run on Mac computers; however, presenters should be aware of compatibility issues across platform such as fonts or multimedia formats. Faculty can also easily turn the PowerPoint presentation into a powerful and interactive teaching tool of ultrasonography for unlimited number of learners by following simple guidelines of using PowerPoint and having minimum resources and technical support for software of voice over presentation, such as Camtasia or Captivate.

Keywords: PowerPoint, Ultrasonography, Presentation skills, Effective teaching.

How to cite this article: Cardwell MS, Aranda JH, Hernandez $\mathrm{R}, \mathrm{Ho} \mathrm{H}$. Empowering Your Presentation Skills. Donald School J Ultrasound Obstet Gynecol 2014;8(1):100-104.

${ }^{1}$ Associate Professor, ${ }^{2}$ Assistant Director, ${ }^{3}$ Lead Analyst, Simulation Application Developer, ${ }^{4}$ Professor, Director

${ }^{1}$ Department of Obstetrics and Gynecology, Paul L Foster School of Medicine, Texas Tech University Health Sciences Center at EI Paso, Texas, USA

${ }^{2}$ Technical Division, Center for Advanced Teaching and Assessment in Clinical Simulation, Paul L Foster School of Medicine, Texas Tech University Health Sciences Center at El Paso, Texas, USA

${ }^{3,4}$ Center for Advanced Teaching and Assessment in Clinical Simulation, Paul L Foster School of Medicine, Texas Tech University Health Sciences Center at EI Paso, Texas, USA

Corresponding Author: Michael S Cardwell, Associate Professor, Department of Obstetrics and Gynecology, Paul L Foster School of Medicine, Texas Tech University Health Sciences Center, 4801 Alberta Ave., EI Paso, TX 79905, USA Phone: 9155456942, e-mail: michael.cardwell@ttuhsc.edu
Source of support: Nil

Conflict of interest: None

\section{INTRODUCTION}

PowerPoint (PPT), a Microsoft proprietary presentation program and ultrasound images work together like a handin-glove. Unlike the days of the $35 \mathrm{~mm}$ slide, PPT has revolutionized technical presentations. ${ }^{1}$ With PPT, preprocessing features allow the contrast, brightness, orientation and annotation to be manipulated before the final slide is produced. ${ }^{2}$ Virtual ultrasound lectures may also be composed using voice-over software such as Camtasia or Captivate. Learners then may view the presentation at their own pace and avoid the usual classroom interruptions and distractions. ${ }^{3}$ With voice-over presentation and screen video capture software, the PPT presentation can be easily transformed into an effective e-Learning tool.

The readers are encouraged to use PPT 2010 or 2013, if available. ${ }^{4}$ The various enhanced functions including the capability to insert online video, image and audio as well as the export presentation as Windows Media Video (WMV) and MPEG-4 (Moving Picture Experts Group) video in PPT 2013 are more user-friendly then previous versions. Cine loops of ultrasound images can be embedded within the slides using animated graphic interchange files (GIFs). The reader is referred to Pomerantz and Choy for their excellent, concise explanation of the procedure. ${ }^{5}$ Most picture archiving and communication systems (PACS) allow the direct importing of the ultrasound images into PPT through joint photographic experts groups (JPEG).

Once the selected ultrasound images or video are imported into PPT, it is very important to remove personally identifiable information (PII) from all media in compliance with Health Insurance Portability and Accountability Act (HIPAA). One easy method to remove identifying information is to use the cropping feature of PPT and/or choose deidentifying option indicated in reporting system or PACS. Other methods are available and the reader is referred to Pomerantz and Choy for these processes. ${ }^{5}$

\section{Simple Guidelines for Effective Use of PowerPoint in Teaching Ultrasonography}

A few simple guidelines are recommended in preparing PPT presentations on ultrasound imaging ${ }^{6}$ : (1) keep the slide simple; (2) minimize text; (3) focus on the ultrasound 
images; (4) title each slide so that the learner can instantly grasp the message; and (5) effective use of video and multimedia.

Annotation is helpful as long as the learners can see the structure of interest and the short label. Transitions from one slide to the next may add interest and aid in the conversational flow of the presentation but also can be distracting. To avoid distractions in your presentation, limit the use of animations, logos, abbreviations, typographical errors and complex slides. ${ }^{7}$

\section{Efficient Use of the Slide Sorter}

A PPT presentation should be a conversation between the presenter and the learner. For a conversation to proceed smoothly and for the learner to comprehend the message, the presenter must convey the message in a logical manner. The slide sorter function of PowerPoint is the tool that allows the presenter to logically arrange the presentation for maximum comprehension. ${ }^{6}$

\section{Practice Tips for PowerPoint Presentations}

The amount and type of practice is presenter-specific. However, some general tips may be helpful. Practice as if a live audience is present; deliver the presentation in a natural, conversational manner. The presentation should be perceived as spontaneous, though rehearsed. The presenter should not rely on rote memory. The slides should be used as cues and not simply read. Remember that the message is from the presenter and that the slides are used to emphasize the message.

The classic technique of rehearsing in front of a mirror is still helpful for some presenters. The actual slides should be used for rehearsing. The slide sorter is an effective tool for timing and practicing the presentation as noted above. As a rule of thumb each slide should take 1-2 minutes of the entire presentation. Allow time in the presentation for questions and possible interruptions.

Practice enough to feel confident but do not wait until the night before the presentation to start. Rehearsing in front of family or friends may be helpful and elicit critical constructive feedback. Digital cameras or smart phones may be used to record the practice sessions and to permit self-critique. If a pointer is to be used during the actual presentation, then practice with a pointer, if available. However, use the pointer sparingly during the live presentation or it may act as a disruption to the learners.

\section{Principles for using the Right Font and Font Sizes}

The use of appropriate font and font size cannot be overemphasized enough in preparing an effective PPT presentation. Here are 8 principles for selecting the right font and font size:

1. Ensure sharp contrast between font and background.

2. Use standard fonts, such as Times New Roman, Verdana or Calibri. If using uncommon fonts, the computer at other places may not have it and can skewing the text.

3. Be consistent: use the Slide Master to select 2 or 3 fonts for the entire presentation.

4. Select right type of font size for readability: for slide title, use font size 40 or bigger and 32 or bigger for body text.

5. Avoid all capital letters.

6. Use different fonts for headlines and bulleted text.

7. Avoid script type fonts.

8. Use italics sparingly.

\section{Selecting the Appropriate Type of Graph to Enhance Quality of the Presentation}

Selecting and using the appropriate chart and graph to represent the data is critically important in enhancing the quality of the presentation as well as helping the audience to quickly capture the meaning of complex data set. We frequently use one or more of the following seven types of graph: ${ }^{8}$

1. Bar graph shows individual values horizontally and is used to display values of multiple components or groups. It is not considered a good choice to track changes over time.

2. Column graph shows individual values vertically; and is used to display values of multiple components or groups. It can be used to track changes over time.

3. Line graph is used to track changes over periods of time. These graphs are useful for finding trends.

4. Pie chart is used to display values of multiple components of a whole. For better display, pie chart should be limited to no more than 5 or 6 components.

5. Area graph is similar to line graph and is used to track changes over time for one or more groups. The graph can show multiple data series at a time.

6. X-Y plot is used to demonstrate relationship between two variables.

7. Histogram is used to display the distribution of discrete or continuous data that are measured on an interval scale.

Other important topics in using appropriate charts and graphs are specified in Table 1:

1. Color of the background and those of the data series must have adequate contrast to be easily seen by the audience.

2. Axes labels and values must be clearly displayed.

3. Title of the graph should focus on the meaning of the data, not the data itself.

4. Legend must be included for proper interpretation of multiple data series. 
Table 1: Indications for appropriate use of graphs in the presentation

\begin{tabular}{|c|c|c|c|c|c|c|c|}
\hline Type of graph & $\begin{array}{l}\text { Continuous } \\
\text { data }\end{array}$ & $\begin{array}{l}\text { Categorical } \\
\text { data }\end{array}$ & $\begin{array}{l}\text { Comparison of } \\
\text { components }\end{array}$ & Relationship & Time series & $\begin{array}{l}\text { Frequency } \\
\text { distribution }\end{array}$ & Multiple groups \\
\hline Pie Chart & & $x$ & & & & & \\
\hline Bar Graph & & $x$ & $x$ & $x$ & & & $x$ \\
\hline Column Graph & & $x$ & $x$ & $x$ & $x$ & $x$ & $x$ \\
\hline Line Chart & $x$ & & & $x$ & $x$ & & $x$ \\
\hline Area Graph & & $x$ & & $x$ & $x$ & & $x$ \\
\hline Histogram & $x$ & & & & $x$ & $x$ & \\
\hline Scatterplot & $x$ & & & $x$ & $x$ & & \\
\hline
\end{tabular}

\section{How to Create a Chart or Graph in PowerPoint}

To create a chart, just click on the Chart icon on any content placeholder in the PPT presentation. One can effortlessly add and edit the data when the chart is created. When the Chart is selected, it is easy to format and edit the chart with the Chart Tools. Presenter can select different chart styles on the Design tab to appropriately represent the data.

Presenter also can use SmartArt graphics to create and insert professional quality diagrams of process, hierarchy, or relationship into the presentation. The SmartArt Design tab allows user to choose from several color options and styles in a gallery with effects that fit with the theme. By right-click on the list and point to Convert to SmartArt and preview a diagram, user can quickly convert an existing PPT that contains bulleted points to SmartArt.

Although numerous animations and special effects are available in PPT, excessive use of these features can cause distraction to the audience. User should limit animations to key points of the presentation in order to emphasize the topics without overwhelming the audience.

To create a chart or graph in PPT 2010/2013, do the following (Fig. 1):

1. In PPT, click the placeholder into which user wants to insert a chart or graph.

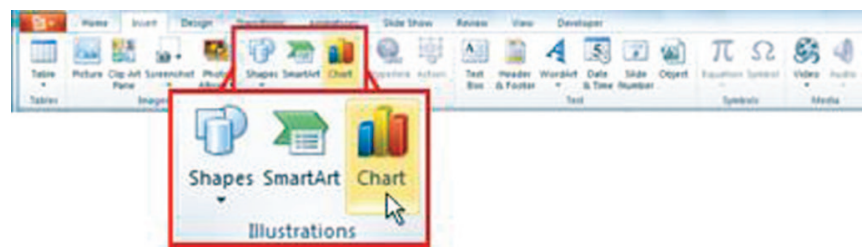

Fig. 1: An illustration to demonstrate a creation of a chart of graph in PPT

2. Do one of the following:

3. In the placeholder, click Insert Chart

4. Click the chart or graph type that user want and click $\mathrm{OK}$.

A Microsoft Excel worksheet with some sample data opens in a new window. To replace the sample data, click a cell on the worksheet and enter the data. User can also replace the sample axis labels in Column A and the legend entry name in Row 1.

5. After entering all data in the Excel worksheet, user clicks the File tab and then clicks Close. The chart in PowerPoint is automatically updated with the new data. User can modify the chart in PPT, including changes to appearance, size or position. Click the chart, then on the highlighted Chart Tools contextual tab, use the Design or Format tab to make changes. To add animation effects to the chart, use tools on the Animations tab.

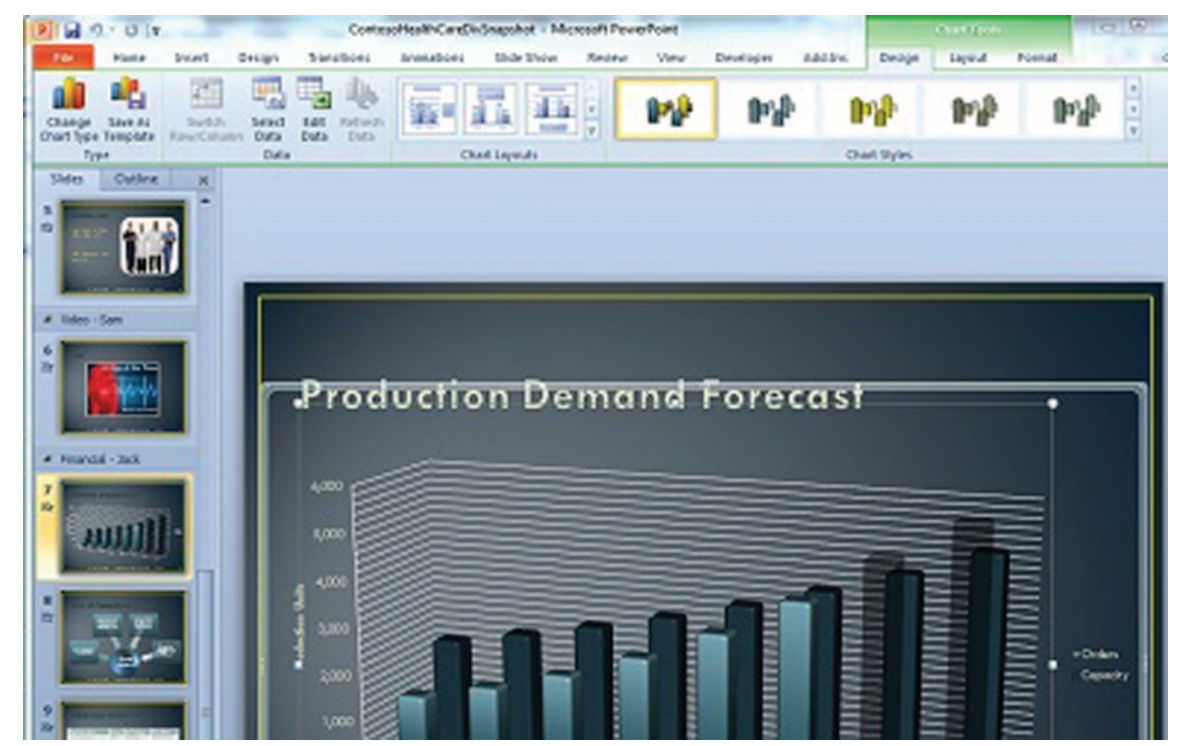

Fig. 2: An illustration how to create a chart using tools on the animations tab 


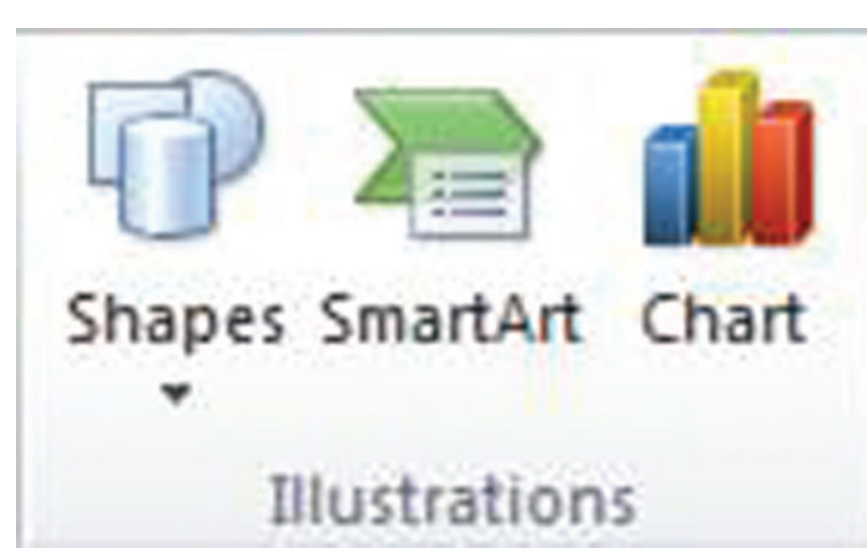

Fig. 3: An illustration how to create a SmartArt graphic

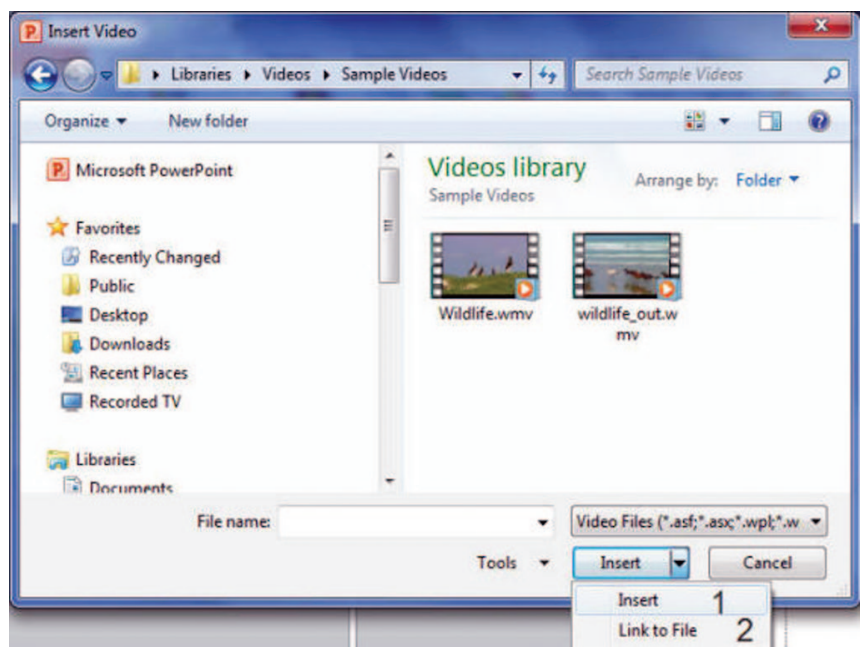

Fig. 4: An illustration how to embed an audio or a video from a file

Similarly, user can edit chart data in PowerPoint by selecting the Design tab, and click Edit Data. For more details, see Change the data in an existing chart.

User can also use SmartArt to quickly create organization charts, flow charts or hierarchical diagram (Fig. 2).

\section{Create a SmartArt Graphic and Add Text to It}

1. On the Insert tab, in the Illustrations group, click SmartArt (Fig. 3).

An example of the Illustration group on the Insert tab, in PPT 2010:

2. In the Choose a SmartArt Graphic dialog box, click the type and layout that user wants.

3. Click (Text) in the Text pane and then type the text.

\section{When do We add Sound or Video to the Presentation?}

In general, we add sound or video to the presentation to highlight an important point and to enhance the audience's understanding that cannot be adequately expressed by the presenter or text (Fig. 4). A recorded narration or a brief video clip that illustrates an important point can be entertaining and help keeping the audience's attention. It's appropriate to inform the audience about the sound or video clip before playing it. PPT 2010/2013 has easy-to-use-tools in which user can customize embedded videos and create effects, fades and video trim. When inserting videos from the files, they become part of the presentation and therefore, eliminate the need for dealing with multiple files.

\section{Embed an Audio or a Video from a File}

1. In normal view, click the slide in which user want to embed a video or an audio clip. ${ }^{9}$

2. On the insert tab, in the media group, click the arrow under video or audio, and then click video or audio from file.

3. In the Insert video-audio dialog box, locate and click the file that user want to embed, and then click insert.

\section{Link to a Video File on a Web Site}

1. User can link to a video file on the local drive or to a video file on a web site, such as YouTube or Hulu. PPT 2010/2013 will automatically embed the desired online video. However, the process for inserting video embedded code is different, and will take additional steps (Fig. 5). For specific instructions, go to Link to a video on YouTube from PowerPoint. ${ }^{9}$

2. It is important that user obtain approval of the material owner before using the link to use or distribute the copyrighted content.

3. On the slides tab in normal view, click the slide to which user want to add a video.

4. In the browser, user can go to the web site that contains the video, such as YouTube or Hulu.

5. On the web site, locate the video and then locate and copy the Embed code.

6. In YouTube, click share, select the use old embed code checkbox, click Embed and then copy the embed code.

7. Back in PPT, on the insert tab, in the media group, click the arrow under video.

8. Click video from Web Site.

9. In the video from Web Site dialog box, paste the embed code and then click Insert.

\section{MICROSOFT POWERPOINT COMPATIBILITY BETWEEN MAC AND WINDOWS}

It's beyond the scope of this article to advise the reader which one is the better platform Mac IOS or PC Windows. However, the reader should be aware of the issues related to PPT between Mac and Windows ${ }^{10}$

1. Use MPEG or AVI on Mac PowerPoint because Quicktime video will not play on Windows PC. 


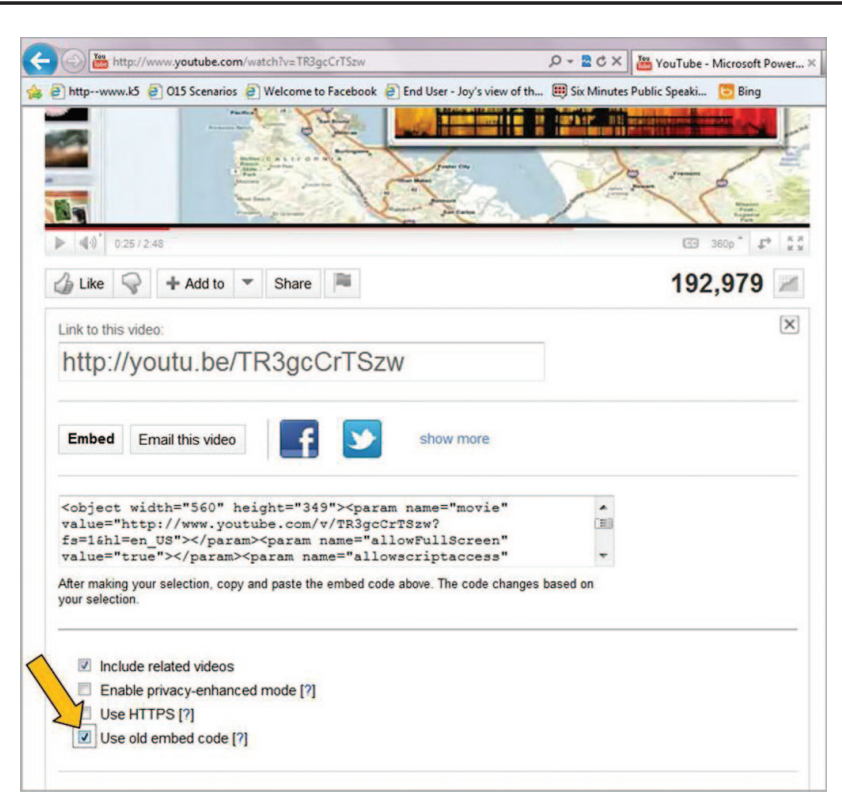

Fig. 5: An illustration how to link a video to a website

2. Use 'Insert Picture' but not 'Copy' and 'Paste' photo into Mac PPT.

3. Embed all graphics because links to external files may break.

4. Use Arial, Times New Roman, Courier and Symbol fonts because they are common on both Mac and PC.

\section{How to Prevent Crash of Your PowerPoint}

PPT is generally a stable program and rarely crashes. However, the user must be aware of the following things to prevent potential crashes of his/her presentation:

1. Avoid playing old PPT files (PPT versions before 2007) on newer PPT.

2. Turn on compatibility mode on PPT $2013 .^{11}$

\section{CONCLUSION}

PPT is a versatile teaching tool for ultrasonography, especially when the users are familiar with features of the software and simple principles of effective presentation. PPT becomes an excellent component of e-Learning when combine with voice-over presentation and screen video capture software such as Camtasia or Captivate.

\section{REFERENCES}

1. Gunderman R, McCammack K. PowerPoint: know your medium. J Am Coll Radiol 2010; 7:711-714.

2. Niamatu J. The power of PowerPoint. Plast Reconstr Surg 2001;108:466-484.

3. Sendra-Portero F, Torales-Chaparro O, Ruiz-Gomez M, MartinezMorillo M. A pilot study to evaluate the use of virtual lectures for undergraduate radiology teaching. Eur J Radiol 2013;82:888-893.

4. Pomerantz S, Choy G. Net assets: PowerPoint pearls for radiology presentations, part II. Radiol 2010;255:687-691.

5. Pomerantz S, Choy G. Net assets: PowerPoint pearls for radiology presentations, part I. Radiol 2010;255:19-22.

6. Dodds C. PowerPoint presentations. Curr Anaesth Crit Care 2004;15:69-73.

7. Harolds J. Tips for giving a memorable presentation, part IV. Clin Nucl Med 2012;37:977-980.

8. Taggert. Brooks representing data graphically. Available at: http:// www.uwlax.edu/faculty/brooks/bus230/handouts/designing\%20 graphs.pdf

9. Embed or link to a video from the presentation. Available at: http://office.microsoft.com/en-us/powerpoint-help/embed-orlink-to-a-video-from-your-presentation-HA010374729.aspx

10. Microsoft PowerPoint compatibility between Mac and Windows. Available at: http://techbar.blogs.brynmawr.edu/1482.

11. Open a presentation created in a different version of PowerPoint. Available at: http://office.microsoft.com/en-us/powerpointhelp/open-a-presentation-created-in-a-different-version-ofpowerpoint-HA102749657.aspx 